\title{
Frontières
}

\section{Danielle Laberge (dir.), L'errance urbaine, Québec, Éditions MultiMondes, 2000, 439 p.}

\section{Diane Laflamme}

Volume 14, numéro 1, automne 2001

URI : https://id.erudit.org/iderudit/1074172ar

DOI : https://doi.org/10.7202/1074172ar

Aller au sommaire du numéro

\section{Éditeur(s)}

Université du Québec à Montréal

\section{ISSN}

1180-3479 (imprimé)

1916-0976 (numérique)

Découvrir la revue

Citer ce compte rendu

Laflamme, D. (2001). Compte rendu de [Danielle Laberge (dir.), L'errance urbaine, Québec, Éditions MultiMondes, 2000, 439 p.] Frontières, 14(1), 77-78. https://doi.org/10.7202/1074172ar

Ce document est protégé par la loi sur le droit d'auteur. L'utilisation des services d'Érudit (y compris la reproduction) est assujettie à sa politique d'utilisation que vous pouvez consulter en ligne.

https://apropos.erudit.org/fr/usagers/politique-dutilisation/
Cet article est diffusé et préservé par Érudit.

Érudit est un consortium interuniversitaire sans but lucratif composé de l’Université de Montréal, l'Université Laval et l'Université du Québec à Montréal. Il a pour mission la promotion et la valorisation de la recherche. https://www.erudit.org/fr/ 
Danielle Laberge (dir.)

\section{L'errance urbaine}

Québec, Éditions MultiMondes, 2000, $439 \mathrm{p}$.

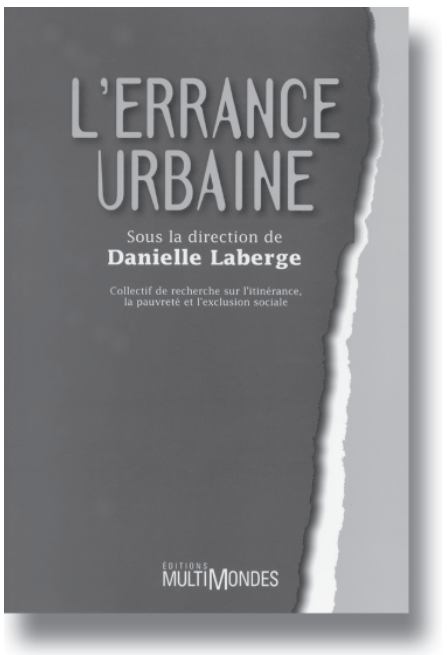

Il faut saluer la parution de cet ouvrage qui nous donne accès aux recherches menées par le Collectif sur l'itinérance, la pauvreté et l'exclusion sociale (CRI). Mis sur pied en 1994 et logé à l'Université du Québec à Montréal, le CRI regroupe des chercheurs rattachés à plusieurs universités québécoises et aussi à divers milieux de pratique et organismes d'intervention sur le terrain. Les données présentées au fil des chapitres nous décrivent des parcours d'autant plus troublants qu'ils sillonnent nos villes, qu'ils croisent nos propres chemins, à Montréal, Québec (chap. 14), Sherbrooke (chap. 10 et 19)...

L'ouvrage a été publié sous la direction de Danielle Laberge, la responsable scientifique du collectif ; elle y propose elle-même trois articles en collaboration avec des collègues et elle signe une introduction et une conclusion où elle réussit, les lecteurs lui en seront reconnaissants, à baliser la démarche et les explorations qui ont suscité les 24 articles répartis dans les deux parties du volume. La table des matières détaillée (elle occupe neuf pages !) a été utilement placée au tout début de l'ouvrage et elle rend moins douloureuse l'absence d'un index. Le lecteur qui connaît peu le phénomène de l'itinérance et qui est à la recherche de données rattachées à une problématique particulière comme les jeunes contrevenants, le système pénal (chap. 7), la pénurie de logements, I'hébergement communautaire (chap. 23), I'appauvrissement des femmes (chap. 5), I'aide alimentaire (chap. 19), les services de santé (chap. 17), peut ainsi facilement repérer les textes susceptibles de lui fournir un éclairage pertinent... et il est fort probable qu'il se laissera vite convaincre d'élargir sa lecture pour remettre en perspective les problèmes qui, à l'origine, l'ont amené à consulter ce volume. Presque tous les articles rappellent, chacun à leur façon, le danger «des découpages thématiques (santé mentale, drogues, prostitution, etc.), négligeant l'entièreté de l'expérience vécue » (p. 83) et l'importance «d'aborder le phénomène de l'errance urbaine comme une problématique d'ensemble où sont inextricablement liées «les formes de construction du lien social et le destin de l'itinérance " (p. 25), comme un " construit » (p. 226, 417), une "production» (chap. 3 ; p. 94, $139,437)$ qui va bien au delà d'un simple « catalogage des pathologies et des handicaps personnels des personnes itinérantes $»(p .2)$.

La table des matières est suivie d'une brève présentation de chacune et de chacun des auteurs. Considérant la variété de l'expérience de travail et des intérêts de recherche des membres du CRI, le lecteur voudra aussi prendre quelques minutes pour situer la voix de l'auteur de l'article consulté. Ainsi, certains articles qui semblent de prime abord en recouper d'autres parce qu'ils abordent un sujet déjà traité dans un chapitre précédent (par exemple, les références à Dernier Recours Montréal aux chapitres 21 et 22) font écho à un vécu, à une chronologie et à des préoccupations qui, grâce à l'expérience des auteurs dans les milieux d'intervention, suscitent des émergences là où I'on aurait pu craindre une répétition.

L'ouvrage s'ouvre sur deux chapitres bien construits et agréables à lire qui nous replongent utilement dans I'histoire. D'abord une « petite histoire " que l'on connaît peu : celle de l'intervention auprès des sansabri à Montréal de 1880 à 1957 (p. 19 à 24). Les auteurs replacent ces données historiques dans un contexte plus général qui vient éclairer les mutations du phénomène complexe qu'est l'errance en milieu urbain. Le deuxième chapitre, écrit par JeanMarc Fontan, élargit le cadre d'analyse en décrivant la "construction historique » de notre conception de l'errance dans la pauvreté, lors du passage de nos sociétés à la modernité (fin du Moyen Âge et début de la Renaissance) et au capitalisme marchand (p. 30-31). Cette réflexion approfondie débouche sur un diagnostic pertinent et, ce qui est plus rare, sur une piste d'intervention qui mise sur la capacité des personnes en cause ; elle mérite d'être longuement citée: "l'arsenal déployé par l'État et la société civile est très important en termes de ressources, mais jusqu'à un certain point ces ressources manquent d'efficacité parce que la rupture des solidarités primaires, une fois qu'elle a atteint un certain niveau de distanciation, ne peut être remplacée par quelques institutions et plusieurs milliers de travailleurs du social. Au problème de dilution du lien social doit correspondre une réponse rapide en termes de recomposition d'une forme de socialité adaptée au problème vécu par l'individu. Cette solution implique forcément une participation des acteurs concernés dans la construction et dans l'actualisation de la réponse " (p. 42-43).

Il est intéressant de quitter provisoirement ce deuxième chapitre pour sauter au quatrième où l'auteur, qui se présente comme un " porteparole des pratiques communautaires", décrit "la fracture sociale entre le monde des travailleurs et celui des sans-emploi dans le contexte québécois" (p. 71). On comprend mieux ensuite l'ampleur vertigineuse de la "solution" proposée au chapitre 2. C'est une solution qui, Jean-Marc Fontan le dit bien, « force la société à un véritable examen de conscience sur le prix qu'elle impose à ses membres pour atteindre une finalité matérialiste dont on ne semble voir la fin ni comprendre I'utilité » (p. 43), une solution qui obligerait les élites actuelles à "redéfinir les bases du contrat social » (p. 44), une solution qui nous demande, à chacune et à chacun d'entre nous, "d'accepter que des entités sociales autonomes puissent vivre et grandir en dehors du cadre normatif du productivisme » (p. 41). Serions-nous en train de nier toute capacité aux personnes que nous jugeons incapables de se mouler aux cheminements récompensés par la logique du capitalisme, aux personnes qui ne sont pas en mesure d'inscrire l'adresse d'un domicile à la ligne prévue à cet effet dans tous les formulaires qui meublent notre quotidien de citoyen ordinaire? Une réflexion s'impose sur le " défi de la citoyenneté » (p. 43-45 et 438-439) dans nos sociétés opulentes et c'est d'ailleurs à cet impératif que Danielle Laberge réserve les derniers paragraphes de la conclusion.

Faisons encore un saut, cette foisci vers des chapitres qui font état des conversations échangées avec ces soi-disant incapables ou qui citent leurs mots. Nous y constaterons notre propre incapacité à décoder les parcours et trajectoires qu'ils inscrivent pas à pas dans "notre" territoire urbain (chap. 6), à usage contrôlé par "nos" règlements municipaux (chap. 16), notre incapacité à comprendre l'histoire de leur désinsertion sociale à l'écart du système de parenté dans lequel nous sommes confortablement "insérés" (chap. 8 et 9), les formes choquantes à nos yeux que prennent leurs efforts de « bricolage identitaire » (chap. 11, p. 214) ou les personnages insolites qu'ils incarnent (chap. 12) soit pour garder la solitude (chap. 13) à distance, soit pour préserver une solitude qui leur est chère. Arrêtons-nous au chapitre 14 pour y lire parmi les trois scénarios proposés celui des «marginaux romantiques» (p. 268) confrontés au système pénal, pour constater comment certaines de nos politiques favorisent "la criminalisation des conditions de vie de l'extrême pauvreté et de l'itinérance » (p. 271), ou encore pour écouter au chapitre 20 comment des « victimes d'un acte criminel " expriment leur peur... des policiers ( $p .373$ ).

Le paradoxe se fait plus envahissant d'un chapitre à l'autre. On ne se surprend donc pas que la deuxième partie de l'ouvrage s'intitule: Paradoxes de l'aide et du contrôle. On y trouve deux chapitres qui tentent une référence timide mais prometteuse à l'approche systémique (chap. 15 et 18). On pourrait souhaiter qu'ils se fassent plus convaincants: Quels sont exactement ces "paradoxes dans les attitudes qui nous empêchent d'agir ensemble" (p. 275) ? Quelles sont ces « répercussions qui frappent les sujets à un niveau phénoménologique » (p. 344) ? La réflexion fondamentale sur le paradigme de la complexité n'est que brièvement évoquée à partir de textes des années 1970 et 1980 (Ackoff, Checkland, De Rosnay, Lemoigne, Morin, Barel) et l'on ne peut que déplorer que les récentes avancées de la systémique (entre autres sur les systèmes autoréférentiels) et de la cybernétique de deuxième génération (l'observation des systèmes-observateurs) soient ainsi passées sous silence. Plus qu'à leur approche théorique, l'intérêt de l'argumentation développée dans ces chapitres est lié au fait qu'ils proposent une description attentive, faite de l'intérieur même du «système de prise en charge de l'inadaptation $»$ (p. 343).

Le volume se termine par un chapitre bien écrit qui permet au lecteur de se familiariser avec l'approche de la réduction des méfaits, le contexte historique de son apparition et les enjeux soulevés par son application. Cette approche, surtout associée à la toxicomanie, est également appliquée "pour tenter de minimiser les effets non désirables reliés à d'autres problèmes sociaux " (p. 421). L'Opération Nez Rouge en est un bon exemple. L'article présente 
efficacement le dilemme auquel sont confrontés les intervenants lorsqu'ils tentent de dépasser la contradiction entre d'une part une "idéologie dominante fondée sur un idéal d'abstinence et sur une politique de criminalisation » (p. 425) et, d'autre part, des pratiques de « gestion des risques » qui se placent " sous le signe du pragmatisme et de I'humanisme » (p. 422-423).

On constate que plusieurs définitions de l'itinérance nous sont proposées d'un chapitre à l'autre de cet ouvrage. Elles viennent préciser divers aspects $d$ 'un phénomène complexe, d'une situation extrême marquée par une souffrance indélébile. C'est par un long processus de mise en retrait, voulue ou subie, qu'un individu et sa société en arrivent ainsi l'un vis-à-vis de l'autre à ce que Jean-Marc Fontan appelle au deuxième chapitre "le terminus de I'indifférence » (p. 36). Les lecteurs qui prendront le temps de parcourir les chemins qu'ont balisés pour lui les chercheurs du CRI seront mieux outillés pour sortir de cette indifférence.

Diane Laflamme 